\title{
Telemetry, Command and Control of UAs in the U.S. National Airspace
}

\author{
Presented By: Barry R. P. Jackson, Cahon Systems Inc. \\ RTCA SC228 Committee Member
}

1.1

Abstract

This presentation summarizes the current draft of the Minimum Operational Performance Standards (MOPS) generated by the Radio Technical Commission for Aeronautics (RTCA). The MOPS will be referenced by the FAA in its Technical Standard Order (TSO) to allow Unmanned Aircraft (UA) to fly in the U.S. National Airspace (NAS).

The TSO permits vendors to certify that their product meets the UA System (UAS) Control and Non-Payload Communication (CNPC) link standard, and if appropriate, the Detect And Avoid (DAA) standard. A TSO certified unit will simplify the certification process.

This presentation deals with the CNPC link only.

\subsection{Introduction and History}

The RTCA organization has supported the FAA for many years in developing DO- $x x x$ type documents. From 2006-2012, the RTCA Special Committee (SC)-203 worked with the FAA to develop the standard to support UA flights within the NAS, including small UASs (sUASs). ${ }^{[1]}$ Using this initial work, the (SC)-203 made a presentation to the International Telecommunications Union (ITU) for a spectrum allocation.

The ITU, at its 2009 meeting, agreed that a protected spectrum for CNPC needed to be allocated; see report ITU-R M.2171. Subsequently an allocation in L and C bands were sanctioned viz:

- L band 960 to $1164 \mathrm{MHz}$ (may not be available for use in the U.S.A. due to a DoD concern with US Navy TACAN interference)

- C band $5030-5091 \mathrm{MHz}$

Because the original scope was too broad, (SC)-203 was disbanded and its effort was not completed. In 2013 the RTCA formed (SC)-228 with a narrower scope, building on the material developed under (SC)-203 and ITU-R M.2171, namely to develop the MOPS needed for non sUASs:

a) UAS DAA system [focus of Working Group (WG) 1]

\footnotetext{
${ }^{[1]}$ The limits placed on small UASs (drone systems) were initially described in the FAA's notice of proposed rulemaking (see the Federal Register, Volume 80; No.35; February 23, 2015) to add a new part 107 to Title 14 Code of Federal Regulations (14 CFR). This addition would "allow for routine civil operation of small UAS in the NAS and to provide safety rules for those operations".
} 
b) UAS CNPC link (focus of WG 2).

Phase 1 of the MOPS being developed by (SC)-228 supports UA flights in the radio Line-OfSight (LOS) terrestrial environment. Phase 2 will cover non-LOS CNPC links. The CNPC link components and interfaces are shown within the rectangle in Figure 1 CNPC link major componentsFigure 1.

The CNPC link is one of several potential data links between any UA and the ground. An example of a non-CNPC link would be mission payload data. These links do not contain safety-of-flight information, and are outside the scope of this presentation.

The CNPC link carries all the data associated with controlling the safe flight of a UA including pilot to Air Traffic Control (ATC) audio communication.

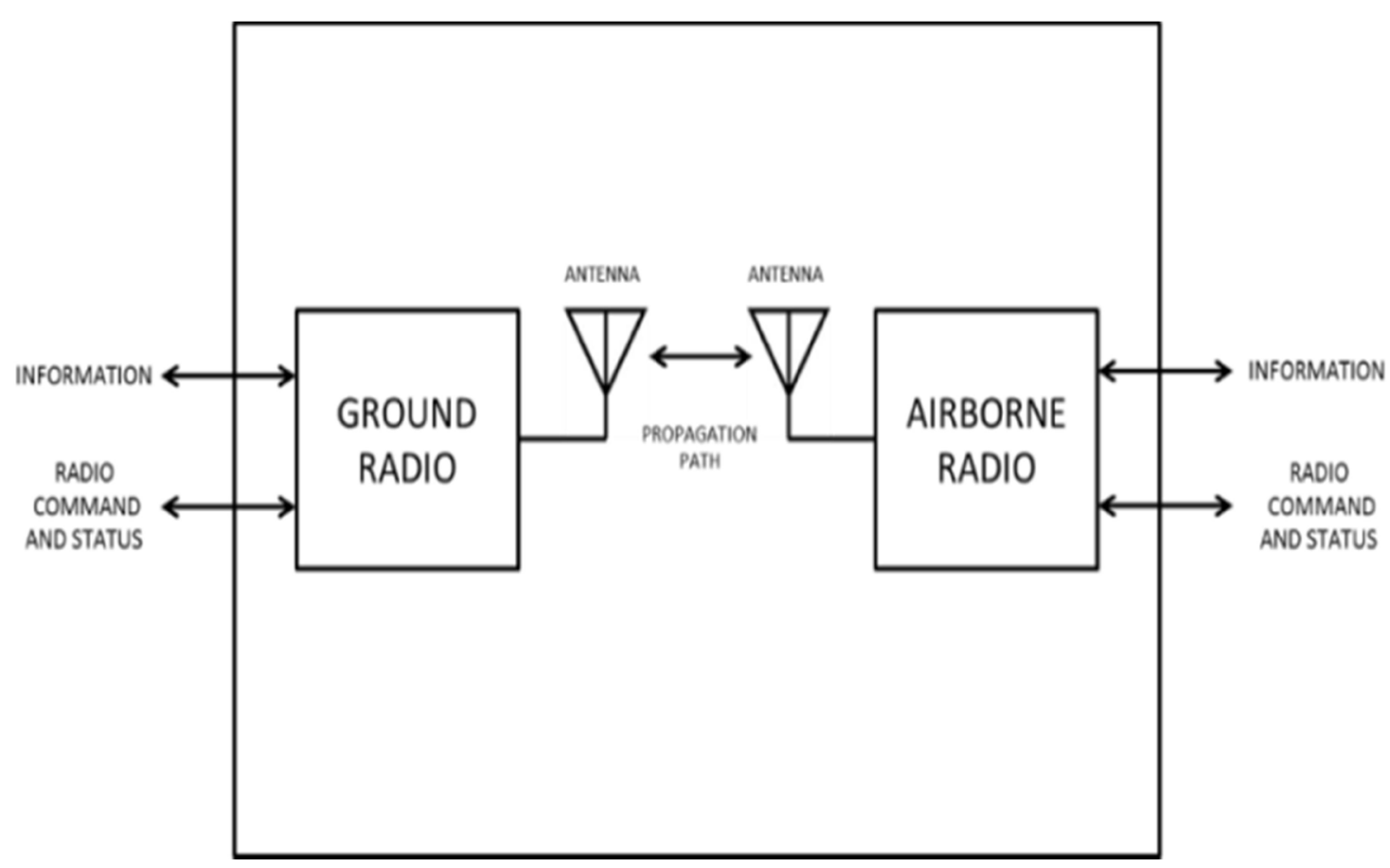

Figure 1 CNPC link major components

To confirm the authority of the MOPS, there will be a thorough validation process utilizing simulations and flight tests using CNPC link prototype airborne and ground radios and antennas. The MOPS will also include verification methods that can be used by radio designers to show compliance of their proposed radio and antenna designs with the MOPS.

The remainder of the presentation will give a brief overview of:

- Operational applications where the CNPC link could be used.

- Specific examples of UA operations.

- Needed information transfers.

- Translating UA operational scenarios into needed CNPC link features.

- System overview. 
- Intended function.

- Anticipated growth of these CNPC link MOPS.

- Annotations and acknowledgements.

\section{3}

\section{Operational Applications}

This section reviews the operational aspects for UASs.

\section{Overview of the Extent of UA Operational Scenarios}

The MOPS developers recognize that there are many possible flight profiles that could be flown by UAs within or among the seven airspace classes defined by the FAA. To help explain the challenges in developing the CNPC link MOPS, three example UA flight paths within the airspace classes are shown in Figure 2.

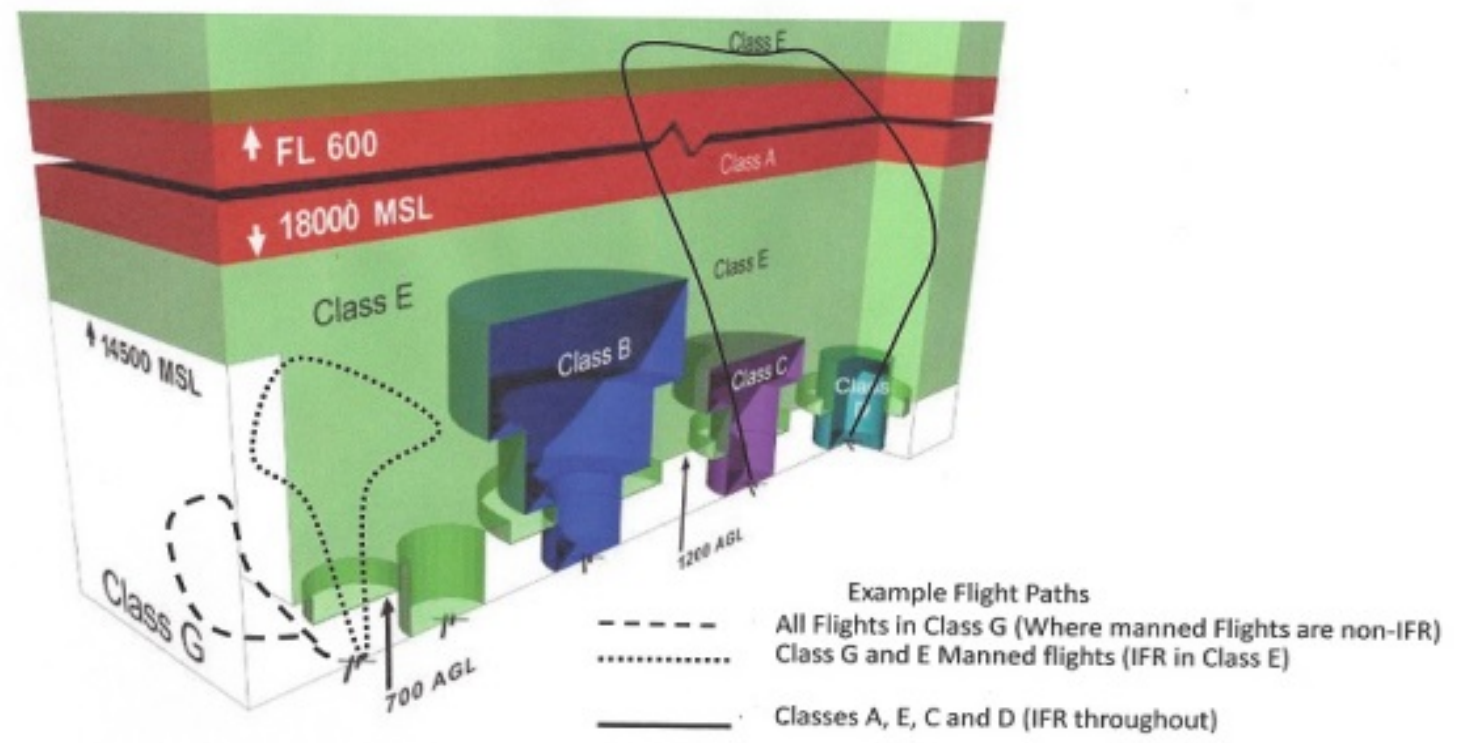

Figure 2 U.S. Airspace Classes with 3 examples of UA flight paths superimposed

The sizes of the UAs, their flight performance, and their avionics and sensor features range from simple to very complex. It is expected that a UA capable of flying within more congested airspace classes would be required to carry additional navigation and communication systems. The Ground Control Station (GCS) used by these UA pilots will be more complex than, for example, a GCS designed for a UA flying within class G airspace.

There are many possible flight paths, and associated types and amounts of information that may need to be sent to/from the pilot to promote safe flights.

There are also many different sizes of UAs coupled with a varying set of complexity of systems/sensors within the UAs and in GCS.

The MOPS is structured to identify standards that would be common to nearly all of the wide varieties of operational flights and types of UAs. When such commonality cannot be found, 
ranges of radio design characteristics that would support selected CNPC link features will be defined in the MOPS.

The current MOPS support flight operations similar to those shown in the dotted and dashed lines shown in Figure 2. This is defined as a point-to-point communication link.

The MOPS will be expanded to address flights where multiple UAs need to communicate with multiple ground terminals as they fly over extended ranges, i.e. flights similar to that shown by the solid line within Figure 2 .

The MOPS describes the design characteristics for a set of equipment that multiple manufacturers could build and field. Those systems would be compatible in that they would not interfere with one another; however, they would not necessarily be interoperable.

In some scenarios, ground CNPC radio systems communicating with multiple UAs (whose CNPC airborne radios are manufactured by different companies) would need to have some degree of interoperability. At this time, there is insufficient data to specify the standards needed to achieve such interoperability.

\section{4}

\section{Specific Examples of UA Operations}

The flight profiles within the following four figures (Figure 3 through Figure 6) depict probable scenarios. The first two figures show examples of a flight path only within class $\mathrm{G}$ airspace. The other two are examples of flight paths classes $\mathrm{G}$ and $\mathrm{E}$ and classes A, E, C, and D from Figure 2.

Figure 3 shows a scenario that would be a near-term practical use of a UA flying in relatively remote areas, yet beyond the current proposed sUAS regulations. The UA is flying beyond the visual line-of-sight of the pilot but not within range of ATC radars. The CNPC link operational requirements include establishing a relay connection(s) that is within radio line-of-sight of the UA.

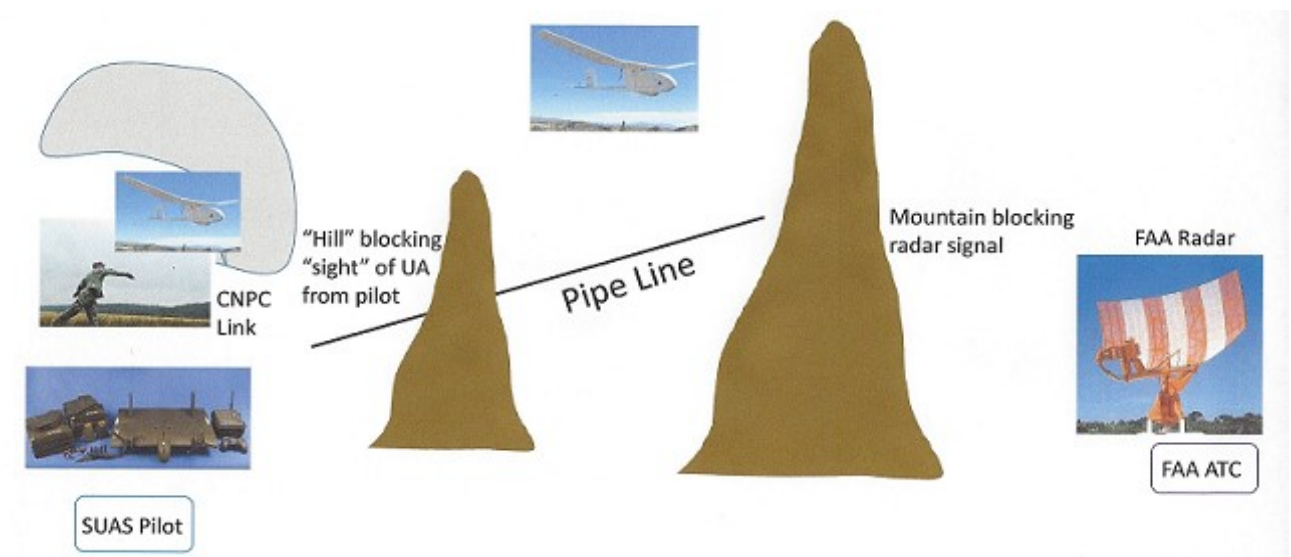

Figure 3 UA following a pipe line in mountains

Another potential operational scenario for sUAS operating outside of the current FAA sUAS regulations is shown in Figure 4; this shows multiple UAs flying in the same area conducting a package delivery or perhaps a video over-watch, all within an urban area. The key additional operational requirements for the CNPC link, beyond that of the above 
example, are to safely handle signals coming to/from multiple UAs, the possible utilization of multiple third parties operating the ground terminal systems, and ensuring continuity of information transfers when transitioning between multiple CNPC link ground terminal system sites.

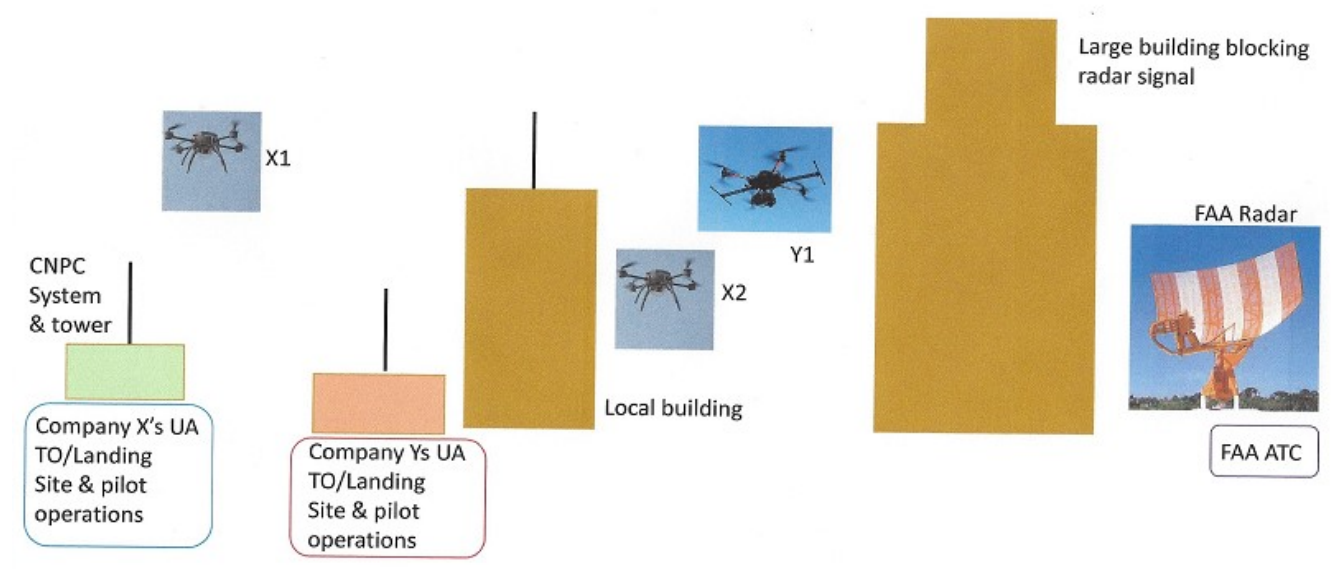

Figure 4 sUAs conducting operations in an urban environment

For flights within class E airspace, (see Figure 5) the CNPC link operational requirement is to ensure the link can carry additional information transfers. This is needed to support safe operation when flying in the class E airspace where many other aircraft are operating; some of those are flying under Visual Flight Rules (VFR) and others under Instrument Flight Rules (IFR). If there is just one UA flying near a ground terminal at any given period of time, then these MOPS would support this scenario. If there are more than one, then the manufacturer would need to develop their own solution (e.g. expanded standards) to address that scenario.

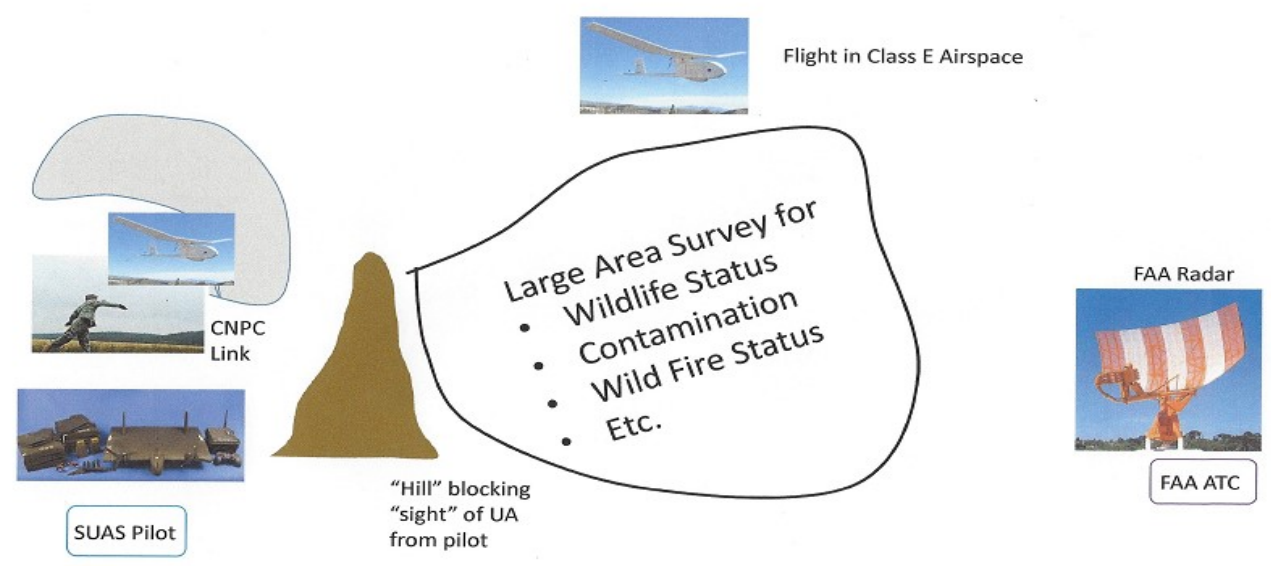

Figure 5 UAs flying in class $\mathrm{G} \& \mathrm{E}$ airspace

The most complex operational scenario is when UAs are flying in class $\mathrm{C}$ and $\mathrm{D}$ airspace and en route through classes A and E airspace (see Figure 6). The key additional CNPC link operational requirement is to ensure the link can carry any additional information transfers 
needed to support safe operation when flying in the class A airspace and to communicate with approach/departure control and airport towers/ground control when within class C or D airspace. Since the MOPS does not explicitly support multiple UAs communication with one ground terminal, manufacturers would need to develop their own solutions to support this scenario.

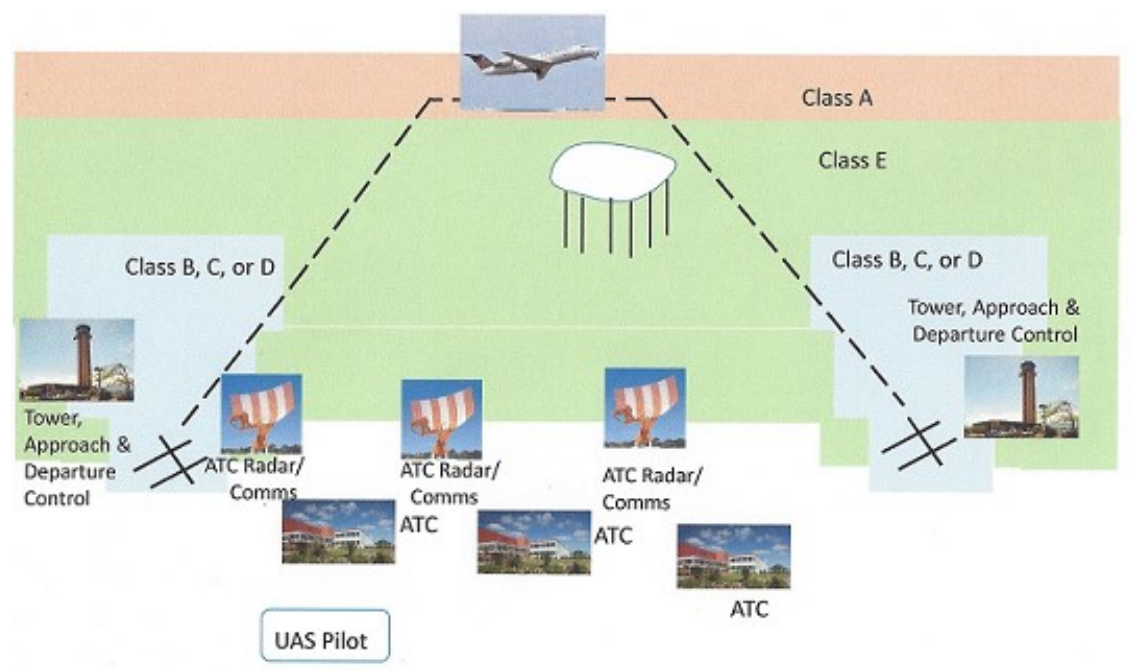

Figure 6 UAs flying in class A, E, C \& D airspace matching those of most commercial airlines

\subsection{Needed Information Transfers Specific Examples of UA Operations}

With the wide range of flight scenarios, there is also a wide range of information transfers that would be needed during specific phases of the flight. During taxiing, takeoff, and landing the pilot would need to be in close contact with the tower, departure, and approach controllers. During taxi, takeoff, and landing, they may also need video from the UA's video camera(s) to keep the UA moving safely along the designated route. This video data sent via the CNPC link will need to be updated often, and need greater bandwidth as it is being sent. However video transmission may not be required throughout flight.

During normal flight, the pilot may need only periodic updates that all is "normal". As such, relatively little data would need to be sent (low bandwidth need), the update rate would be low, but would need to occur on a regular basis. During normal flights it is to be expected that the pilot would change some of the preset flight path guidance uploaded to the UA prior to takeoff, e.g. changing waypoints or altitudes to be flown. In this case, there would be an increase in the uplinked data to the UA. The data transmitted would be greater than during normal flight; the data would need to be updated often to confirm receipt and ensure the UA follows the new guidance.

However, in adverse weather conditions, when flights near other aircraft (when DAA actions may be required) or during emergency situations, the amount of data to be sent from the UA would increase. Video and/or weather data may be required to be sent to the pilot, 
and corresponding commands from the pilot to the UA. As such, a greater bandwidth would be needed, but this would not normally occur often during a flight.

A summary of these information transfers is displayed in Figure 7.

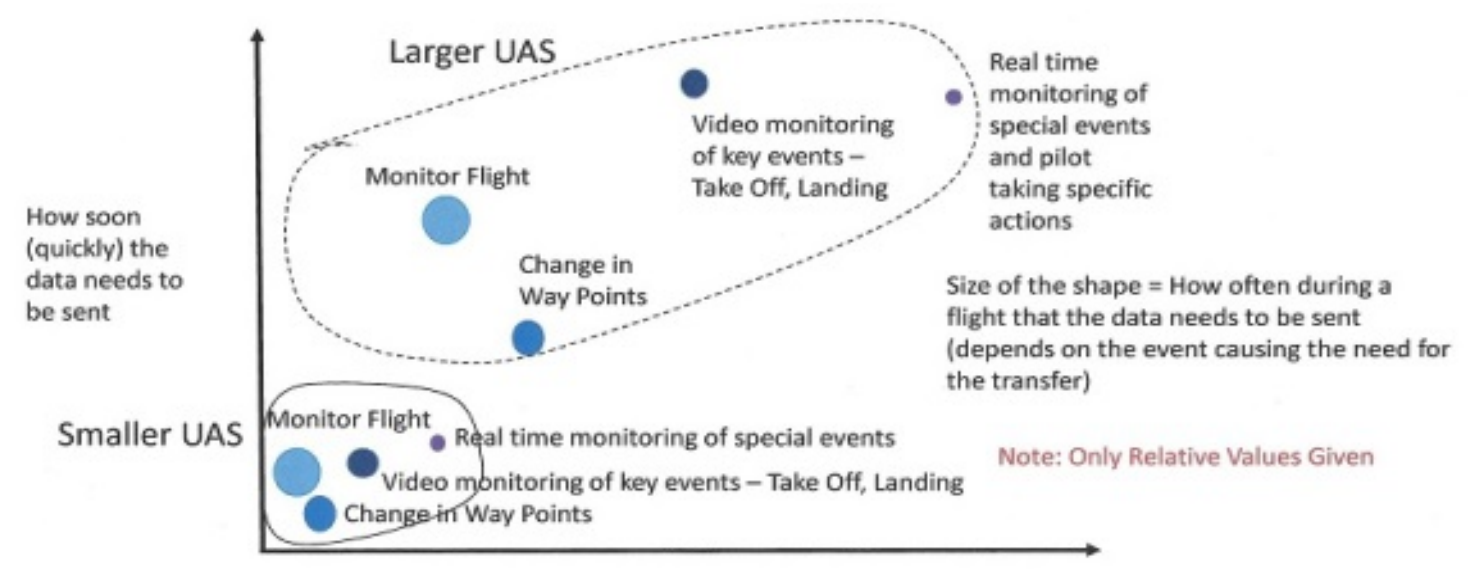

Amount of data in transfer for a particular exchange

Figure 7 Relative Information Transfers that could be needed during UA flights in the NAS

Once the operational requirements and the types of information transfers are determined, the next step is to define the features of the CNPC link so it can support such operations.

Note: only general descriptions of the key features are identified; the details of these and the specific design characteristics requirements will be in the final MOPS. The required features are:

- Support transfers of flight control information to/from pilot and UA, e.g. actual pilot control and monitoring of the UA's flight.

- Support for information transfers for such features as

- UA location/navigation information, e.g. GPS, VOR, DME.

- Detect And Avoid.

- Voice and data communications with airport towers, arrival and departure controlers, ATC, ATC radars, etc.

- Video images during taxiing, takeoff, landing, and emergencies.

- Weather information, e.g. data from a weather radar on the UA.

- Status information of key systems on the UA (to include the CNPC link airborne radio) and confirmation of changes to those systems. 
- Data from selected backup/redundant systems on the UA.

- A Pilot is In Command (PIC). A Pilot is on the loop at all times. No autonomous flight is allowed.

- UA flights follow IFR requirements (FAA mandated if a flight is within the NAS). The CNPC link supports the needed information transfers for compliance.

In addition to the above, there are several features based on current FAA needs and regulations:

- The CNPC link airborne antenna(s) supports effective propagation to CNPC ground radios during all possible UA maneuvers.

- The CNPC airborne radio does not cause interference with other electronic systems on the UA (of particular concern is possible interference with transponders), in the area where the CNPC link ground terminal is located, or around other systems within the "radio horizon" of the CNPC link airborne antenna.

- Spectrum utilization needs to be both efficient and effective so it can eventually support a large number of UAs operating in the same area simultaneously.

- Sufficient spectrum is required to support any needed CNPC link redundancy to ensure the needed information transfers reach a satisfactory performance and safety level.

- The signal modulation selected must support changes in the bandwidth needed during normal UA flights, during emergency situations and when multiple UAs are flying in the same general area.

- The information sent over the CNPC link will be encrypted and authenticated to provide resistance to potential interference.

The current MOPS does not explicitly require the features italicized above; they may be addressed in a future development effort (Phase 1A MOPS).

\section{7}

\section{System Overview}

For successful UAS operations in the NAS, there are many systems beyond the scope of the CNPC link MOPS. A typical representation of these systems and the interfaces between those systems is illustrated in Figure 8. The key elements of the CNPC link are shown in solid lines and squares; systems outside the link are represented by dashed lines and squares.

\section{Basic System}

Within Figure 8, the systems outside the CNPC link MOPS include:

On the ground side:

- A distribution system to connect to and from the ground side of the CNPC link to the ground Flight and Radio Management System (FRMS).

- The FRMS to connect to and from the pilot's cockpit. 
- A link from the pilot's cockpit to and from a Spectrum Authority (which assigns the frequencies that the CNPC link will use at various times and locations).

- A link from the pilot's cockpit (either directly and/or relayed through the UA) to ATC so flight clearances can be given and acknowledged.

On the air side:

- The airside of the CNPC link is connected to and from the aircraft's FRMS.

- The FRMS links to and from the various flight control, avionics, and other systems on the UA.

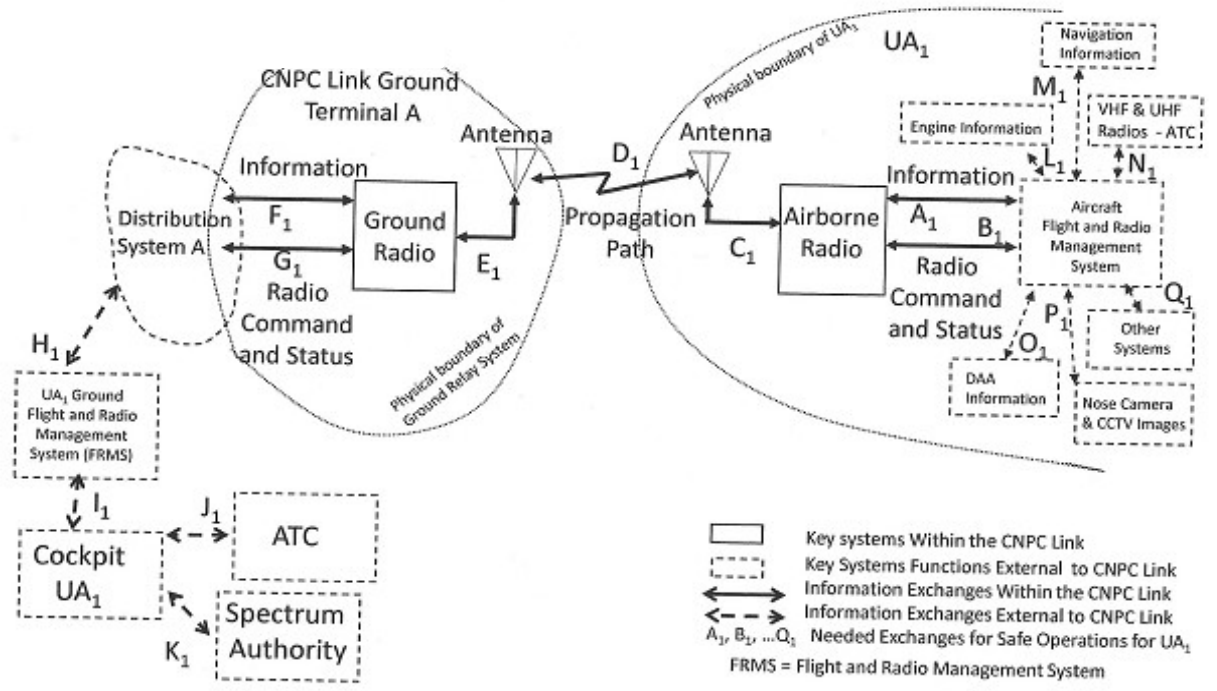

Figure 8 Basic Information transfers within \& outside the CNPC link needed for safe UAS operations within the NAS

\section{Basic CNPC link Functions}

The MOPS describes the features and characteristics needed to achieve a point-to-point communication function for UAs flying in the NAS. There are additional features and characteristics that may be needed, but not included in this MOPS, to achieve multi-point communications when multiple UAs are seeking to communicate with a single ground terminal.

Depending on the intended operations, the CNPC link equipment and interfaces described will also support one or more of the following features of a point-to-point communication function:

- ATC voice and data relay

- DAA data exchange 
- Weather radar data exchange

- Video data exchange

All of the equipment shall comply with the appropriate Federal Communications Commission (FCC) rules and International Civil Aviation Organization (ICAO) Remotely Piloted Aircraft Systems (RPAS) Standards. It will also provide a means to follow appropriate ATC procedures for UAs flying in the NAS.

Characteristics having defined values for all terrestrial CNPC links are listed in Table 1.

Table 1 Characteristics having defined values for all point to point CNPC links.

\begin{tabular}{|l|l|}
\hline R-f & Frequency stability \\
\hline Power rise time and fall times & Frequency quanta (smallest radio channel width) \\
\hline Command and status interfaces & Output power and emission mask \\
\hline Frequency bands to be used (L- and C-Bands) & Adjacent channel and spurious response rejection \\
\hline Waveform structure [Time Division Duplex (TDD)] & Compatibility with other systems \\
\hline
\end{tabular}

In addition there are characteristics that are deemed to be manufacturer dependent, i.e., the manufacturer could select specific design characteristic values that best meet the radio manufactures chosen market sector and are given in Table 2 .

Table 2 Characteristics having variable values for all point to point CNPC links.

\begin{tabular}{|l|l|}
\hline Symbol rate & Receiver Sensitivity \\
\hline Modulation Type & Frequency Capture Rate \\
\hline Transmitter Output Power & Doppler Correction \\
\hline
\end{tabular}

\section{Environmental Functions}

Unless otherwise specified, the environmental conditions contained in RTCA DO-160G, (or later revision) will be used to establish the airborne equipment environmental standards criteria. The environmental conditions contained in RTCA DO-160G, and MIL-STD-810G, will be used to establish the ground equipment standards.

\section{Assumptions}

It is assumed that radio manufacturers have sufficient technical knowledge to understand the MOPS and experience to combine the various equipment described within the scope of the MOPS. With that background multiple venders should be able to manufacture the equipment. It is understood that the MOPS do not fully define all of the design characteristics but they are the minimum necessary for such developments. 
- As noted in several subsections, this MOPS does not include all of the design characteristics for the CNPC link to support multiple UAs communicating with just one ground terminal radio.

- For single ground CNPC radio systems to successfully communicate with multiple UAs whose CNPC airborne radios were manufactured by different companies, there needs to be some degree of interoperability between the ground radios and each airborne radio. Once sufficient information and knowledge is gained, and there is sufficient need for such a capability, the FAA could request the RTCA to develop such a MOPS.

- In addition, the SC-228 TOR already identifies the requirement to develop MOPS to support UA CNPC link communications via satellites. A separate MOPS development team will be required for that effort.

The current MOPS is in draft form and will be sent out for consultation; thus the foregoing is subject to change.

This presentation is a compilation of the original work by members of the RTCA SC228. The diagrams and some of the text are RTCA copyrighted and are used with their permission. The complete document will be available for purchase via the RTCA website (or at the address below) mid 2016 when the final document will be released.

RTCA, Inc.

$115018^{\text {th }}$ Street NW

Suite 910

Washington, DC 20036

(202) 833-9339

www.rtca.org 